\title{
Une critique du mensonge par-delà le bien et le mal
}

Eine Kritik der Lüge jenseits von Gut und Böse

A critique of lying beyond good and evil

Jochen Mecke

\section{CpenEdition}

\section{Journals}

Édition électronique

URL : http://journals.openedition.org/ceg/1701

DOI : $10.4000 /$ ceg. 1701

ISSN : 2605-8359

\section{Éditeur}

Presses Universitaires de Provence

Édition imprimée

Date de publication : 1 décembre 2014

Pagination : $91-110$

ISSN : 0751-4239

Référence électronique

Jochen Mecke, « Une critique du mensonge par-delà le bien et le mal », Cahiers d'Études Germaniques [En ligne], 67 | 2014, mis en ligne le 17 décembre 2017, consulté le 04 novembre 2020. URL : http:// journals.openedition.org/ceg/1701 ; DOI : https://doi.org/10.4000/ceg.1701 


\title{
Une critique du mensonge par-delà le bien et le mal
}

Jochen MECKE

Université de Regensburg

\begin{abstract}
Il ne saurait y avoir de doute : aucune pratique linguistique ou sémiotique ne se trouve être condamnée avec tant d'insistance, tant de mépris et tant d'émotion que le mensonge. Chaque fois qu'un sportif, un chercheur, un journal ou un homme politique est accusé de mensonge, la condamnation est unanime et une véritable déferlante médiatique s'abat immédiatement sur la personne en question. Les exemples sont légion : G. W. Bush et Colin Powell pour les prétendues armes de destruction massive en Irak, l'ancien ministre Cahuzac pour ses comptes cachés en Suisse, et puis la kyrielle de déclarations de gouvernements de tout bord, pour leurs fausses promesses pré- ou postélectorales, du type : "Il n'y aura pas de suppressions d'emplois chez Areva », «Les rétrocommissions de Karachi : une fable ! ", "Si la gauche gagne, nous rétablirons la retraite à 60 ans ", « Les hausses d'impôts ? Jamais tant que je serai président! » etc., etc. ${ }^{1}$ Dans tous ces cas de mensonges politiques, les tempêtes médiatiques déclenchées montrent bien que la condamnation du mensonge est beaucoup plus radicale et catégorique que celle d'autres pratiques sémiotiques ou linguistiques pourtant aussi graves, comme les insultes publiques, les dénonciations, les atteintes à la vie privée ou bien les campagnes médiatiques orchestrées contre certains personnages publics. Parfois, on a l'impression que le mensonge est jugé d'une manière beaucoup plus sévère que le délit qu'il essaie de cacher $^{2}$. Ainsi, le fait que Jérôme Cahuzac dispose de comptes en banque en Suisse était considéré comme une pratique assez courante et presque acceptée, mais c'est pour avoir nié publiquement et devant le Parlement posséder de tels comptes à l'étranger qu'il a déclenché le véritable scandale.
\end{abstract}

\footnotetext{
${ }^{1}$ Pour une liste des mensonges politiques des dernières années, cf. la documentation rassemblée par Jean-François KAHN, Menteurs !, Paris, Plon, 2012.

${ }^{2}$ Gaspard DHellemmes, « Cahuzac : vers un délit de parjure à l'américaine ? », Le Journal du Dimanche, 5 avril 2013
} 
Pourquoi cette condamnation catégorique du mensonge ? À première vue, les motifs semblent évidents. Car, à la différence d'autres pratiques ou attitudes linguistiques ou sémiotiques répréhensibles et socialement sanctionnées, le mensonge comporte un aspect négatif supplémentaire, puisqu'il semble détruire la confiance mutuelle et, partant, la cohésion sociale. Il n'est donc pas étonnant que le reproche le plus efficace que l'on puisse adresser à un homme politique ne consiste pas à lui faire grief d'avoir des idées erronées sur les possibilités de faire baisser le chômage ou bien de ne pas savoir comment fonctionne l'économie du pays, mais à l'accuser de mentir aux électeurs, vu que ce reproche détruit non pas sa compétence, mais sa crédibilité. Il ne s'en prend pas à une simple erreur momentanée, mais à l'être même de l'adversaire politique, car il ne relève pas du domaine du savoir ou du savoir-faire, mais de la morale. Ainsi, lors des élections présidentielles de 2012, le journal Le Point titrait avec grand succès «Qui ment le plus? » pour trancher entre les deux candidats Nicolas Sarkozy et François Hollande.

Ces effets du mensonge semblent également la cause d'une longue tradition de désapprobation à son endroit. L'un des commandements du décalogue ne dit-il pas : «Tu ne mentiras point? » Et même des courants de pensée en principe opposés au dogmatisme théologique, comme la philosophie des Lumières, dont on peut espérer un jugement désintéressé, pesant le pour et le contre au-delà de la morale chrétienne, sont unanimes à condamner le mensonge; le point culminant de ce rejet est sans doute l'interdiction de mentir absolue et catégorique prononcée par Immanuel Kant, même au cas où le mensonge pourrait sauver la vie d'une victime. Les parents, quant à eux, n'enseignent-ils pas à leurs enfants de ne pas mentir ?

Cependant, cette vision du mensonge est peut-être trompeuse car, d'un autre côté, les statistiques montrent que le commun des mortels ment en moyenne deux fois par jour ${ }^{4}$; dans certaines expériences menées par des psychologues, le chiffre s'élève à trois mensonges en dix minutes si les personnes ne se connaissent pas, et selon d'autres recherches, nous mentons à un interlocuteur sur trois ${ }^{6}$, tandis que d'autres encore affirment qu'on nous ment à peu près deux cents fois par jour ${ }^{7}$. Indépendamment du bien-fondé de telles statistiques, force est de constater que tout le monde ment et, qui plus est, que les mêmes citoyens qui condamnent leurs hommes politiques avec

\footnotetext{
${ }^{3}$ Le Point, $\mathrm{n}^{\circ}$ 2058, 23 février 2012.

${ }^{4}$ Bella M. DEPAOLO et al., « Lying in everday life », Journal of Personnality and Social Psychology, n 70, 1996, p. 979-995.

${ }^{5}$ Robert FelDMAN et al., « Self-Presentation and Verbal Deception : Do Self-Presenters Lie More ? ", Journal of Basic and Applied Social Psychology, vol. 24, n 2, 2002, p. 163-170.

${ }^{6}$ DEPAOLO, « Lying », p. 979-995.

${ }^{7}$ James GEARY, « How To Spot A Liar », Time Europe, vol. 155, n 10, 13 mars 2000, (http://www.time.com/time/europe/magazine/ 2000/313/lies.html [12 avril 2014]). La différence entre toutes ces statistiques s'explique évidemment en partie par les différentes acceptions du mensonge. Ainsi, la définition de Geary semble très large et inclut des pratiques comme la politesse, qui ne font pas partie du mensonge selon d'autres définitions. Nous y reviendrons.
} 
véhémence considèrent les petites tricheries de la déclaration d'impôts comme quelque chose de tout à fait normal, mentent à leurs conjoints ou conjointes et ne disent pas la vérité à leurs enfants à qui ils enjoignent pourtant de ne jamais mentir. Quelles conséquences faut-il en tirer ? Peut-on, comme le suggèrent certains auteurs, avoir une attitude décomplexée par rapport au mensonge ? Car lorsqu'on passe en revue à la fois les derniers scandales qui ont marqué les sociétés allemande et française et les récentes publications plutôt populaires sur l'éthique du mensonge, on ne peut qu'être frappé par une contradiction entre théorie et pratique éthiques : alors que les journaux, les informations télévisées et les blogs sur Internet ne cessent de dénoncer le mensonge comme le grand fléau de notre société, des articles et des livres souvent consacrés à la pratique quotidienne du mensonge semblent être marqués par une relativisation considérable de cette condamnation. Ainsi, la journaliste Claudia Meier prône le mensonge dans son livre Lob der Lüge. Warum wir ohne sie nicht leben können ${ }^{8}$, se faisant l'écho de Peter Stiegnitz ${ }^{9}$, «mentiologue » auto-proclamé qui est allé jusqu'à développer une théorie appliquée du mensonge dans Lügen - aber richtig! Die angewandte Theorie der Lüge ${ }^{10}$, un ouvrage qui défend et enseigne, comme son titre l'indique, le droit au mensonge et invite ouvertement à mentir chaque fois qu'on en a besoin. Le dernier exemple est le livre tout récent du germaniste Wolf Schneider intitulé Die Wahrheit über die Lüge. Warum wir den Irrtum brauchen und die Lüge lieben ${ }^{11}$.

Compte tenu de ces contradictions, la seule certitude est que nous retrouvons dans notre attitude envers le mensonge la même ambiguïté que celle qui caractérise le mensonge lui-même. C'est peut-être pour cette raison que depuis deux mille ans, pour le monde occidental, le mensonge n'a rien perdu de sa fascination. L'intérêt général pour le personnage du menteur, tels Ulysse, le héros picaresque du roman Lazarillo de Tormes, les imposteurs comme Tartuffe, Tonio Kröger ou Bardolino, la séduction exercée par Pinocchio, qui ment du reste très peu, et le succès de séries télévisées comme Lie to $m e^{12}$ prouvent que le mensonge exerce depuis toujours une attraction considérable sur la culture occidentale.

Cela soulève de multiples questions. Pourquoi une société, des institutions comme l'Église catholique, des partis politiques, l'État et des individus condamnent-ils avec autant de radicalité ce qu'ils pratiquent eux-mêmes aussi

\footnotetext{
${ }^{8}$ Claudia MAYER, Lob der Lüge. Warum wir ohne sie nicht leben können, Berlin, List, 2007.

${ }^{9}$ Peter StiEgnitz, Lügen lohnt sich. Lüge, Wahrheit, Wirklichkeit, Frankfurt am Main, Haag \& Herchen, 1991.

${ }_{10}$ Peter STIEGNITZ, Lügen - aber richtig! Die angewandte Theorie der Lüge, Wien/ Klosterneuburg, Va Bene, 2008.

${ }^{11}$ Wolf SCHNEIDER, Die Wahrheit über die Lüge. Warum wir den Irrtum brauchen und die Lüge lieben, Reinbek bei Hamburg, Rowohlt, 2012. Du côté francophone, Marie-France Cyr arrive au même constat : Marie-France CYR, La vérité sur le mensonge, Montréal, Éditions de l'Homme, 2003.

12 Brian GraZer, David Nevins, Samuel Baum, Lie to me, USA, Fox, 2009-2011.
} 
souvent? Est-ce un exemple de plus de la différence classique entre théorie et pratique, exigence morale et comportement réel, soi-même et les autres, qui nous fait condamner chez autrui un comportement que nous acceptons tacitement pour nous-mêmes ? Ou bien y aurait-il à ce refus des raisons spécifiques, qui relèveraient de la structure même du mensonge ?

Pour répondre à ces questions, il faut essayer de jeter un regard étranger et étrange sur le mensonge, et en particulier s'abstenir de tous les jugements préalables suggérés par la tradition judéo-chrétienne occidentale. L'on doit tenter d'examiner le mensonge à partir d'une perspective se situant au-delà du bien et du mal afin d'analyser son véritable fonctionnement au sein de la société. Ce parti pris nous permettra également de décrypter non seulement les fonctions du mensonge, mais aussi celles de sa condamnation catégorique. En somme, ce que nous allons entreprendre ici correspond d'une certaine manière à une « critique du mensonge $»^{13}$.

Pour ce faire, il faut relever trois défis : d'abord, le défi éthique, pour savoir s'il y a une éthique du mensonge en général. Mais ce défi est intimement lié à un autre, d'ordre sémantique, car le jugement éthique porté sur le mensonge dépend évidemment de la définition de ce dernier. En dernier lieu, il s'agit de relever un défi culturel, celui de savoir pourquoi une société condamne aussi catégoriquement d'un côté ce qu'elle pratique aussi fréquemment de l'autre. Notre hypothèse est que cette contradiction, ou bien cette ambiguité, est directement liée à la structure et la fonctionnalité du mensonge même.

\section{Éthiques du mensonge}

L'histoire de l'éthique occidentale est marquée par une condamnation catégorique du mensonge qui peut se ramener à l'interprétation que l'Église catholique a donnée au huitième commandement : ainsi, saint Augustin, pour qui le mensonge revêt une importance tellement capitale qu'il y consacre deux monographies, De mendacio (395) et Contra mendacium (420), paraît se borner à reprendre ce commandement et à le justifier par une série d'arguments supplémentaires. Certes, saint Augustin développe, pour la première fois, toute une typologie et une casuistique du mensonge, mais pour finalement condamner tous les mensonges d'une manière catégorique et indifférenciée ${ }^{14}$. Déjà, saint Augustin discute l'exemple célèbre du mensonge adressé à un malfaiteur pour protéger sa victime potentielle. Selon ce Père de l'Église, même dans ce cas-là, un mensonge n'est point permis car, par ce mensonge mortel, nous sacrifions notre propre vie éternelle et céleste ${ }^{15}$, tout

\footnotetext{
${ }^{13}$ Il s'agit ici d'une critique au sens extra-moral du terme, qui essaie d'examiner les fonctions, les pouvoirs et les limites du mensonge.

${ }^{14}$ Aurelius Augustinus, Die Lüge und Gegen die Lüge, trad. par Paul Keseling, Würzburg, Augustinus-Verlag, 1953, p. 11-16.

${ }^{15}$ Ibid, p. 32 .
} 
en protégeant la vie temporaire et terrestre de la victime. Plus tard, Kant, à l'occasion d'un débat avec Benjamin Constant et se plaçant évidemment dans une perspective tout à fait différente, rejoindra saint Augustin dans la condamnation absolue, car il pense qu'un mensonge contrevient au droit de tout homme, et de l'humanité entière, à la véracité ${ }^{16}$. Le Père de l'Église catholique et le philosophe des Lumières, en dépit de leurs avis généralement opposés, sont donc unis dans la conviction que le mensonge est entièrement répréhensible. Depuis la Bible, en passant par saint Augustin et Kant, pour arriver à ce qui semble être, en ce moment, le discours socialement reconnu sur le mensonge, nous trouvons donc une condamnation générale et de principe, la seule différence consistant en l'acceptation ou non des exceptions qui permettent de mentir dans quelques cas bien particuliers.

Mais quand on élargit un peu l'horizon pour prendre en considération d'autres cultures et d'autres temps, il apparaît clairement que cette condamnation de principe est loin de faire l'unanimité. Dans l'Iliade et l'Odyssée, le rusé et menteur Ulysse est à l'honneur et se trouve loué par une déesse, même quand il ment apparemment sans nécessité aucune. C'est Pallas Athéna elle-même qui approuve les ruses et mensonges de son protégé, car comme elle le souligne - il ressemble à la déesse ${ }^{17}$. On pourrait objecter à cet argument qu'il est tout à fait relatif car il semble que la littérature ne fasse ici que plaider sa propre cause. Mais même Platon, qui selon la vulgate voudrait pourtant exclure les poètes de la Cité parce qu'ils mentent ${ }^{18}$, considère le mensonge comme légitime pour le chef d'État ${ }^{19}$.

Néanmoins, quand on examine les textes antiques de plus près, il s'avère que la Bible elle-même est loin de rejeter le mensonge de manière catégorique. Certes, de nombreux passages le condamnent, mais les Saintes Écritures font preuve envers lui de la même ambiguïté que la société actuelle. Ainsi, en de nombreux endroits, l'Ancien Testament raconte des mensonges sans commentaires ni jugements, surtout quand ces mensonges sont dus à des personnages en principe positifs, considérés de surcroît comme des ancêtres et des fondateurs du peuple juif. C'est par exemple le cas de Jacob, qui ment à son père Isaac pour prendre la place de son frère, et se faire ainsi bénir et choisir comme héritier ${ }^{20}$. Le mensonge peut également recevoir l'approbation divine, comme le montre l'exemple des sages-femmes juives qui mentent au pharaon et sont récompensées par Dieu ${ }^{21}$. Il arrive même à Jésus de mentir,

\footnotetext{
${ }^{16}$ Immanuel KANT, « Über ein vermeintes Recht aus Menschenliebe zu lügen », in Immanuel Kant, Werke in zehn Bänden. éd. par Wilhelm WeISCHÄDEL, Darmstadt, Wissenschaftliche Buchgesellschaft, 1984, p. 637-643, ici p. 35 sq.

${ }^{17}$ HomèRe, Odyssee, trad. par Johann Heinrich Voss, München, Goldmann, 1980, vers 290-299, p. 156 sq.

${ }^{18}$ PlAton, « Der Staat » in Sämtliche Dialoge, t. V, traduit et commenté par Otto APELT, Hamburg, Meiner, 1988, p. 404.

${ }^{19}$ Ibid., p. 91. Avec cette exigence, Platon anticipe les justifications du mensonge qu'un Machiavel développera plus tard à la Renaissance.

${ }^{20}$ La Bible, Ancien Testament, Genèse 27, 1-39.

${ }^{21}$ La Bible, Ancien Testament, Exode, 1, 15-20.
} 
comme lors de la fête juive des cabanes, quand il commence par annoncer qu'il n'y assistera pas, pour finalement y prendre part quand même ${ }^{22}$. Certes, Dieu hait le mensonge et ne saurait mentir lui-même, mais il envoie néanmoins un « esprit de mensonge » au roi Achab pour l'induire en erreur et lui faire perdre une bataille ${ }^{23}$. Et le fameux huitième commandement n'ordonne pas, comme on le croit d'ordinaire : « Tu ne mentiras point !», mais «Tu ne témoigneras pas faussement contre ton prochain ${ }^{24}{ }^{4}$. Le huitième commandement ne condamne donc pas le mensonge en tant que tel, mais son usage pour nuire à autrui, par exemple lors d'un procès. Ainsi, force est de constater que ce qui semble déterminer le discours dominant toute une tradition occidentale - la condamnation du mensonge en tant que tel, indépendamment des effets qu'il peut produire - est loin d'être une évidence quand on regarde d'autres temps et d'autres cultures. Au contraire, tout porte à croire que la condamnation du mensonge en soi et indépendamment de ses objectifs, effets et conséquences est une attitude bien propre à la tradition chrétienne et catholique, qui paraît remonter à saint Augustin et dont les motifs demandent une explication.

\section{Pour une définition extra-morale du mensonge}

Saint Augustin semble être le véritable fondateur du discours occidental sur le mensonge au sens foucaldien du terme, non seulement parce que l'évêque d'Hippone a, le premier, consacré deux monographies au mensonge, mais aussi parce qu'il jette les bases d'un changement de paradigme dans la définition et la théorie du mensonge ${ }^{25}$. "Ainsi donc mentir», écrit-il, «c'est avoir une chose dans l'esprit, et en énoncer une autre soit en paroles, soit en signes quelconques. ${ }^{26}$. Le changement par rapport à Platon et à la philosophie grecque ne saurait être plus radical.

1. En plaçant le mensonge dans «l'âme » ou dans « l'esprit », Augustin sépare nettement le mensonge de l'erreur. Ainsi, la mesure du mensonge n'est plus la vérité, mais l'opinion ou la conviction personnelles. C'est là procéder par subjectivisation et intériorisation du mensonge.

\footnotetext{
${ }^{22}$ La Bible, Nouveau Testament, Évangile selon saint Jean, 7, 2-10.

${ }^{23}$ La Bible, Ancien Testament, Rois, 1, 22, 22, TOB, Paris, Éd. du Cerf/ Les Bergers et les Mages, 1987, p. 673. Du point de vue littéraire, il s'agit là évidemment d'un simple transfert métonymique.

${ }^{24}$ La Bible, Ancien Testament, Exode, 20, 16, TOB, Éd. du Cerf/ Les Bergers et les Mages, 1987, p. 167

${ }^{25}$ Cf. Luc-Thomas Somme, «La vérité du mensonge », Revue d'Éthique et de Théologie morale, 2005, HS, $\mathrm{n}^{\circ} 236$, p. 33-54.

${ }^{26}$ Augustinus, Lüge, chap. 3, p. 2 sq. ; tr. française, Saint Augustin, « Du mensonge », in Euvres Complètes, traduction de M. l'abbé DevolLle, traduites pour la première fois en français sous la direction de M. RAULX, t. XII, Bar-le-Duc, L. Guérin, 1866, p. 195-217, ici p. 196.
} 
2. De ce fait, Augustin fait sortir le mensonge du domaine de la connaissance et de l'épistémologie pour le placer uniquement dans le domaine de la volonté humaine, et donc de l'éthique. Dorénavant, le mensonge consiste en une contradiction entre «opinion »- et non pas vérité - et «expression». En cela, Augustin s'oppose clairement à la théorie de Platon qui avait distingué deux types de pseudos («mensonge ») : le premier, qu'il appelle également le "vrai mensonge» ou «le mensonge de l'âme » et qui correspond à l'erreur, lui paraît beaucoup plus condamnable que le second, qu'il nomme le «mensonge par des paroles » et qui correspond au mensonge au sens propre du terme $^{27}$. Avec saint Augustin, le contraire du mensonge n'est plus la vérité, mais la véracité. Dans sa réponse à $\mathrm{B}$. Constant, Kant précise la nécessité d'une telle distinction, car la vérité n'est pas assujettie à notre volonté, elle ne saurait lui être soumise, puisque nous pouvons toujours nous tromper quand nous affirmons quelque chose, tandis que nous sommes tout à fait libres de décider si nous voulons dire ce que nous considérons comme la vérité ou non. Ce qui relève du domaine de notre volonté n'est donc pas la vérité, mais notre conviction ou notre opinion. Contrairement à la vérité, la véracité ou la sincérité peuvent faire l'objet de notre décision, nous avons le choix de dire ou de dissimuler ce que nous pensons. Le véritable antonyme du mensonge n'est donc pas comme le suggère notre usage quotidien du mot - la vérité, mais la véracité $^{28}$.

3. Le troisième élément du discours augustinien sur le mensonge a également déterminé la tradition occidentale, mais, cette fois-ci, en la chargeant d'une lourde hypothèque. Car, pour le Père de l'Église, le mensonge se fait toujours dans le but de tromper l'autre. Dans le $5^{\mathrm{e}}$ chapitre de De mendacio il écrit : "Une déclaration fausse [ici il faut comprendre "tenue pour fausse", n. d. J. M.] qui est faite dans le dessein de tromper est un mensonge. $\left.{ }^{29}{ }\right\rangle$

Cette « voluntas fallendi $»$ se trouve encore de nos jours dans la plupart des définitions du mensonge, non seulement dans le catéchisme catholique ou dans la théologie morale, mais également chez des auteurs contemporains. Or, ce « dessein de tromper », qui constitue l'un des rares consensus de la théorie du mensonge, doit son existence à une métonymie discursive qui fait subrepticement passer un élément de la structure du mensonge pour son objectif principal. Car si le mensonge consiste en une divergence entre ce que l'on pense ou ressent d'une part et ce que l'on exprime de l'autre, il va de soi que cette divergence doit rester cachée pour que le mensonge puisse fonctionner. Mais s'il est vrai que la dissimulation de la différence entre

\footnotetext{
${ }^{27}$ PLATON, « Der Staat », p. 84.

${ }^{28}$ KANT, « Über ein vermeintes Recht », p. 36.

${ }^{29}$ Augustinus, Lüge, chap. 5, p. 7.
} 
opinion et expression est un élément nécessaire du mensonge, cela ne veut pas dire qu'elle en soit forcément l'objectif. Si nous opérons une distinction entre la dissimulation de la divergence entre opinion et énonciation d'une part, et les différents objectifs que peut avoir cette dissimulation de l'autre, il apparait clairement que le mensonge peut servir une multiplicité d'objectifs, dont la tromperie constitue seulement une possibilité parmi d'autres. Certes, Augustin ne nie pas que le mensonge puisse poursuivre d'autres buts que celui de tromper, mais pour lui, celui de tromper est toujours et déjà donné.

Des réflexions précédentes, il ressort que le mensonge consiste en un acte linguistique ou bien sémiotique caractérisé par les points suivants ${ }^{30}$ :

1. Dans chaque mensonge, il y a une divergence entre ce que l'on pense et ce que l'on dit, c'est-à-dire entre opinion et énonciation ou bien sentiments et expression.

2. Cette divergence est dissimulée.

3. La dissimulation de la divergence entre opinion et expression peut servir différents objectifs qui, en général, restent également cachés.

Cette définition entraîne plusieurs conséquences, dont la première a déjà été évoquée. Elle consiste en une distinction nette entre l'erreur et le mensonge, deux notions que le mot grec de pseudos incitait encore à confondre. Notre définition réduit donc l'ambiguité sémantique de la notion de mensonge et fait définitivement sortir le mensonge de l'épistémologie pour le placer dans le domaine de l'éthique. Or, c'est justement dans le domaine de l'éthique et de la morale que la définition entraîne un changement de perspective radical. Car si nous avons éclairci le statut de la tromperie et mis en évidence qu'elle ne constitue point l'objectif du mensonge mais se réfère uniquement à la dissimulation de la divergence entre opinion et expression, il s'ensuit que le mensonge, une fois libéré de la relation nécessaire avec le but de tromper, peut devenir le moyen de réaliser une multiplicité d'objectifs différents. Le jugement moral sur le mensonge ne saurait donc être émis de manière catégorique, mais - toujours dans la terminologie de Kant - de manière "hypothétique ", c'est-à-dire en fonction des objectifs dont le mensonge est le moyen de réalisation. Si l'on peut, grâce au mensonge, atteindre un nombre considérable d'objectifs différents, le jugement moral sur le mensonge dépend uniquement de ces objectifs. Certes, un mensonge peut servir à tromper autrui ou bien à ruiner sa renommée, mais il peut également être employé pour inspirer confiance en soi à quelqu'un ou lui donner de l'espoir ${ }^{31}$, pour encourager un ami ou bien - c'est le cas le plus spectaculaire, discuté déjà par Augustin, et plus tard par B. Constant et Kant - pour sauver des vies. Ce jugement non-catégorique mais relatif ou hypothétique porté sur

${ }^{30}$ Cf. Simone DiEtz. Die Kunst des Lügens. Eine sprachliche Fähigkeit und ihr moralischer Wert, Reinbek bei Hamburg, Rowohlt, 2003, p. 25.

${ }^{31}$ Cf. Jakob der Lügner (Jurek BECKER, Frankfurt am Main, Suhrkamp, 1969). 
le mensonge correspond par ailleurs à notre pratique dans la vie quotidienne, où nous évaluons les mensonges en fonction des buts que nous poursuivions.

De ce fait, le contraire du mensonge, la sincérité, perd également le statut d'attitude irréprochable qu'elle avait jusque-là. Avec la sincérité, on peut, certes, créer de la confiance, renforcer l'amitié et la confidence, mais on peut aussi détruire l'estime de soi de quelqu'un, en lui disant par exemple ce que l'on pense vraiment de ses compétences, ou encore agresser autrui, toujours en faisant croire que tout ce qui nous intéresse, c'est d'être absolument sincère ou bien - pour reprendre l'exemple cher à saint Augustin, Kant et B. Constant - dénoncer une victime et être complice d'un assassinat. Walter Benjamin a, dans une esquisse pour un travail sur le mensonge, dénoncé le caractère problématique de la sincérité de certaines personnes qui racontent sans gêne tout ce qu'elles pensent ou ressentent, en assimilant ce comportement à un manque de retenue, de souci de soi et même d'hygiène personnelle $e^{32}$.

Si notre définition nous permet donc de libérer le mensonge de l'idée erronée qu'il est toujours lié à un dommage pour autrui et d'ouvrir une voie pour un jugement plus différencié et équitable en fonction des objectifs que l'on veut atteindre grâce à lui, elle permet également une plus grande précision et un discernement accru dans l'usage du mot. En fait, certains chiffres exorbitants dans les statistiques sur le mensonge reposent sur un manque de précision sémantique. La définition augustinienne, par exemple, devrait en toute rigueur inclure les compliments dans le mensonge, car toutes les conditions fixées par celle-ci s'y retrouvent, à savoir une contradiction entre opinion et expression et le dessein de tromper l'autre ; nous ne voulons certainement pas, en effet, que ce dernier sache que nous pensons le contraire de ce que nous venons de dire et que nous trouvons en vérité la nouvelle coiffure de notre amie horrible. Pourtant, dans la vie quotidienne, personne ne considère les compliments et les formes de politesse comme un mensonge. $\mathrm{La}$ raison en est évidente : tout le monde sait qu'il s'agit là non pas d'une expression de nos pensées personnelles, mais plutôt de formules auxquelles nous obligent les règles de la bonne conduite. Elles obéissent d'une manière évidente et visible pour tous à un code imposé. Mais si un mensonge est annoncé et attendu, il lui manque l'élément de la dissimulation et il n'en est donc plus un. Ce qui annonce ici le mensonge, c'est le code de la communication même, à savoir une situation concrète qui nous signale qu'un énoncé n'est pas l'expression d'une opinion personnelle, mais qu'il est dû au respect des exigences de la situation.

Il en est de même pour toutes les formes de communication indirecte où le contexte ou des signes spécifiques indiquent qu'il s'agit d'un usage « impair» (Frege) de la langue - comme pour les métaphores, les hyperboles,

\footnotetext{
${ }^{32}$ Walter BENJAMin, « Notizen zu einer Arbeit über die Lüge », in Walter BENJAMIN, Gesammelte Schriften, t. VI, éd. par Rolf TIEDEMANN et Hermann SCHWEPPENHÄUSER, Frankfurt am Main, Suhrkamp, 1985, p. 62-64.
} 
les métonymies et tous les tropes et autres figures de rhétorique. Ici, ce sont les signes littéraires eux-mêmes qui révèlent au destinataire du message qu'il $\mathrm{y}$ a une divergence entre opinion et expression et que le message n'est pas à prendre au pied de la lettre, mais au sens figuré. Un cas peut-être encore plus courant dans la vie quotidienne est celui de l'ironie. Si son emploi est facile entre amis, c'est parce qu'en cette circonstance, tout le monde connaît les opinions véritables de celui qui parle et que la contradiction entre ses convictions et un énoncé ponctuel est d'emblée visible. Mais avec des inconnus, il est beaucoup plus malaisé de rendre l'ironie perceptible. La tâche de révéler la divergence entre opinion et expression revient alors à la forme de l'expression elle-même. Ainsi, des figures rhétoriques, des tropes ou bien une prosodie ou une tonalité différentes doivent signaler au destinataire de ne point prendre l'énoncé au premier, mais au second degré. Dans tous ces cas de figure, la divergence entre opinion et expression n'est pas dissimulée, mais manifestée grâce à des signes supplémentaires.

C'est pour cette même raison que la littérature - et ce malgré le soupçon général que Platon a formulé contre elle dans La République - n'est pas susceptible de mentir. En effet, là aussi, le mensonge est annoncé et ne saurait plus fonctionner comme tel. Friedrich Nietzsche a insisté sur cette caractéristique non mensongère de tout $\operatorname{art}^{33}$. L'art et la littérature exposent donc volontairement le caractère inventé et fictif de leurs créations, ils dénoncent leur propre mensonge et ne sauraient donc point mentir, du moins pas au sens propre du terme.

\section{Les fonctions du mensonge}

Nous avons jusqu'ici décrit la structure du mensonge. Mais quelles sont les fonctions du mensonge pour les individus et pour la société ? Pourquoi exerce-t-il une fascination si grande ? Et pourquoi les sociétés et cultures occidentales condamnent-elles d'une manière si véhémente ce qu'elles pratiquent avec autant d'insistance?

Pour l'individu, le mensonge est certainement une technique classique pour apparaître comme ce qu'il n'est pas. En effet, ce que les romans racontent à titre d'exceptions remarquables, c'est-à-dire les faits et méfaits d'imposteurs célèbres, tels Guzman de Alfarache, Martin Guerre, le lieutenant de Köpenick, Mr. Ripley ou Felix Krull, est en réalité une pratique quotidienne. Ainsi, le psychologue Robert Feldman a pu constater lors d'une expérience que des personnes qui ne se connaissent pas mentent en moyenne deux ou trois fois pendant un entretien de dix minutes. L'objectif de ces

\footnotetext{
33 «Kunst behandelt den Schein als Schein, will also gerade nicht täuschen, ist wahr » (Friedrich NIETZSCHE, « Nachlass 1869-1874 », in Kritische Studienausgabe, éd. par Giorgio COLli et Mario MONTINARI, München, dtv, 1999, p. 632 sq).
} 
mensonges est chaque fois le même : se montrer sous un jour flatteur, pour se faire apprécier davantage de l'interlocuteur ${ }^{34}$.

Cette technique consistant à simuler un statut social, une force ou une compétence supérieurs, n'est en fait que la manifestation spécifique d'une fonction plus générale du mensonge, qui sert à compenser des faiblesses ou une position sociale subalterne. Il existe toute une tradition dans laquelle le mensonge est un moyen de se soustraire aux normes et aux valeurs dominantes et à leur contrôle permanent sans risquer toutefois de sanction immédiate. Même si le prince, selon une longue tradition de théorie politique qui s'étend de Platon à Machiavel, a le droit, contre les adversaires de l'État, d'employer le mensonge, celui-ci était en réalité avant tout un instrument des subalternes, des dominés et opprimés, car il leur permettait d'échapper à la surveillance du pouvoir en place. Comme le montre le roman picaresque, la ruse, la simulation, la dissimulation et le mensonge sont surtout des techniques de survie des " hommes infâmes ", sans pouvoir ni moyens de subsistance. Ils servent à déjouer le contrôle d'un pouvoir souverain qui ne laisse aucun « jeu », aucun espace à ses sujets. En particulier, il est un moyen classique dans des situations où le pouvoir exige de ses sujets une adhésion inconditionnelle aux dogmes et à l'idéologie dominante : la dissimulation des convictions et des sentiments ainsi que le mensonge sont les seuls moyens de survie pour les subalternes. Ainsi, l'obligation faite aux juifs et aux musulmans espagnols, soit de quitter le pays, soit de se convertir, crée une injonction paradoxale qui aboutit à un mensonge structurel - "une conversion forcée de bon gré" - provoquant de son côté un soupçon généralisé de mensonge dirigé contre tous les convertis, et contre lequel on ne saurait se défendre que par des mensonges supplémentaires ${ }^{35}$. De la sorte, la surveillance systématique et la délation généralisée créent une structure dans laquelle le mensonge est ancré d'emblée et où le mensonge individuel n'est qu'une réaction particulière à une situation mensongère en elle-même.

Dans une note intitulée "Notizen über "Objektive Verlogenheit" ", W. Benjamin a introduit une nouvelle catégorie ou bien un nouveau type de mensonge qui permet, entre autres, de décrire la particularité de la situation évoquée ci-dessus ${ }^{36}$. Benjamin appelle «mensonge objectif» un type de mensonge qui ne réside plus dans l'individu et où ce dernier n'a aucune responsabilité personnelle, mais est plutôt de bonne foi, tandis que le cadre dans lequel se place cette sincérité est lui-même mensonger ${ }^{37}$. Dans ce cas de figure, la sincérité individuelle et subjective se situe dans un contexte de

\footnotetext{
${ }^{34}$ Robert FELDMAN et al., « Self-presentation and Verbal Deception: Do Self-Presenters Lie More? », in Basic and Applied Psychology, n ${ }^{\circ}$ 24, p. 163-170. Cf. également Robert FELDMAN, Lügner. Die Wahrheit über das Lügen, Berlin/ Heidelberg, Springer Spektrum, 2012.

${ }^{35} \mathrm{Cf}$. Bartolomé Bennassar, L'Inquisition espagnole. $X V^{e}-X I X^{e}$ siècles, Paris, Hachette, 2009.

36 Walter BENJAMIN, " Notizen über "Objektive Verlogenheit" ", in BENJAMIN, Gesammelte Schriften, t. VI, p. 60-62.

${ }^{37}$ Ibid., p. 60.
} 
mensonge objectif et collectif. Pour bien expliquer à quoi Benjamin se réfère, il est peut-être utile de mentionner aussi le cas contraire, où le mensonge individuel se transforme en sincérité objective. Il en est ainsi dans une situation où l'on arrive, grâce à un mensonge subjectif, à révéler une véracité objective, par exemple quand nous mettons quelqu'un à l'épreuve en nous accusant nous-même d'un méfait d'une manière mensongère pour permettre à cette personne de pardonner, ce qui n'aurait pas été possible sans cette astuce $^{38}$. Cela est également le cas quand nous essayons, par un mensonge, de construire un piège pour que quelqu'un avoue ses véritables opinions ou pour qu'il énonce une vérité dissimulée. Certes, le texte de Benjamin, par son caractère fragmentaire, reste à plusieurs égards énigmatique. Mais nous trouvons d'autres indices pour mieux comprendre son approche si nous tenons compte d'une distinction supplémentaire. En fait, comme Hannah Arendt qui différencie les vérités de fait et les vérités de raison ${ }^{39}$, Benjamin établit une distinction entre une " véracité » relative aux faits ("Wahrheit/ Falschheit in den Angaben ») et une sincérité qui se réfère à une vérité supérieure («Wahrheit/ Unwahrheit») $)^{40}$. Or, cette distinction donne lieu à une combinatoire intéressante : de même qu'une fausseté des faits, selon Benjamin, est tout à fait compatible avec une sincérité supérieure, la véracité des faits l'est avec un mensonge supérieur. Benjamin illustre sa thèse par l'exemple de l'éducation sexuelle des enfants, où la véracité des faits peut quelquefois correspondre à un mensonge supérieur, simplement parce que certains faits ne sont pas encore intéressants ou compréhensibles pour un enfant d'un certain âge et vice-versa ${ }^{41}$. Le même chiasme est à l'œuvre quand, dans le cadre d'un régime autoritaire, des insurgés ou des rebelles mentent pour vaincre un système politique mensonger lui-même et imposent de cette manière une vérité supérieure, tandis que la sincérité serait ici un mensonge objectif, car elle serait suicidaire et s'inscrirait dans le cadre d'un système objectivement mensonger ${ }^{42}$. De la même manière, un juif converti qui opterait, dans le contexte de l'inquisition espagnole, pour la sincérité en avouant avoir toujours pratiqué sa religion d'origine est certainement sincère sur le plan subjectif, mais si nous plaçons cette sincérité dans la situation d'oppression et d'extermination des autres religions, il se transforme en mensonge objectif, car les juifs ont été forcés à se convertir «de bon gré». L'affaire Lewinsky illustre un autre cas de figure : dans le contexte des accusations contre Bill Clinton, à qui l'on reprochait d'avoir eu des rapports sexuels avec la stagiaire Monica Lewinsky, l'affirmation du président américain disant «I had no sexual relations with that woman » était, certes, un mensonge subjectif, mais proféré dans une situation qui consistait à

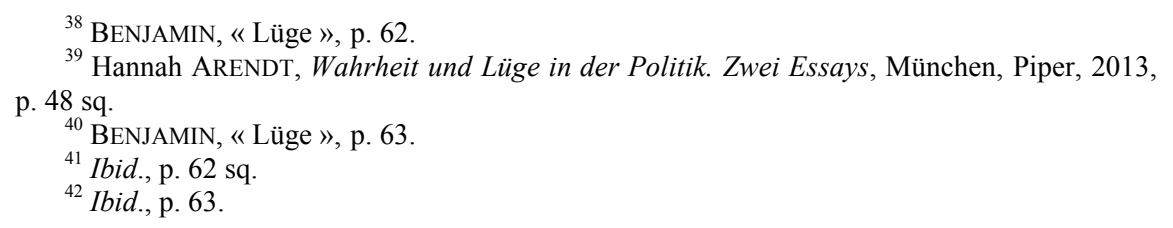


introduire dans le domaine de la politique des critères moraux s'appliquant uniquement à la vie privée. Le mensonge individuel de Bill Clinton servait donc à se protéger contre une ingérence illégitime dans sa vie privée et était de ce fait plus légitime que les accusations dirigées contre lui. Par son mensonge, Bill Clinton défendait une vérité supérieure, la protection de la vie privée de chaque individu, conférant de cette manière au mensonge subjectif une véracité objective supérieure aux accusations soi-disant morales et en réalité mensongères.

Cependant, à première vue, la théorie du mensonge objectif pourrait apparaître comme un retour à la confusion platonicienne entre mensonge et erreur, car dans les deux cas, la conviction subjective est fausse. Mais à la différence de l'erreur platonicienne, le mensonge objectif benjaminien est marqué par une divergence entre l'opinion et l'expression des souverains ou des dominants. Ainsi, les inquisiteurs espagnols savaient parfaitement que leurs accusations servaient, la plupart du temps, des intérêts particuliers, car les délateurs agissaient souvent par envie, antipathie personnelle, rancune ou bien dans le dessein de se débarrasser d'un concurrent ${ }^{43}$. Quant aux inquisiteurs eux-mêmes, leur motivation était souvent d'ordre économique, car l'Inquisition devait se financer au moyen des fortunes confisquées aux 《hérétiques ${ }^{44}$. Rien d'étonnant à ce que beaucoup d'hommes riches aient figuré parmi les accusés. La différence entre l'erreur chez Platon et le mensonge objectif chez Benjamin réside donc dans le fait que chez le philosophe allemand, la divergence ne concerne pas l'individu, mais le souverain ou bien la société entière.

Benjamin rompt donc avec la limitation individualiste et subjectiviste de la théorie du mensonge et prend en considération la diversité et la multiplicité des fonctions qu'il peut avoir dans la société. L'une de celles-ci est en relation directe avec les processus de différenciation dont est issue la société moderne. Car si cette dernière peut se décrire, selon Niklas Luhmann, comme un ensemble de sous-systèmes auto-poïétiques relativement autonomes qui fonctionnent selon leurs propres règles, aucun individu ne sera plus capable de développer des compétences dans tous les domaines, mais chacun se voit forcé de faire appel à des spécialistes à qui il est contraint de faire confiance. La confiance mutuelle joue donc un rôle très important, car elle contribue à réduire la complexité d'une société différenciée et permet d'agir même là où un individu normal n'a pas les données nécessaires pour prendre une décision rationnelle ${ }^{45}$. Or, dans ce contexte, le mensonge est évidemment contreproductif car, s'il est employé à plusieurs reprises, il subvertit les bases de la confiance et donc également celles de la société moderne.

\footnotetext{
${ }^{43}$ Henry Kamen, La Inquisición española. Mito e historia, Barcelona, Crítica, 2013, p. 39, passim.

${ }^{44}$ Henry Kamen, The Spanish Inquisition. An historical revision, London, Weidenfeld \& Nicolson, 1997, p. 153

${ }^{45}$ Niklas Luhmann, Vertrauen. Ein Mechanismus der Reduktion sozialer Komplexität, Stuttgart, Lucius \& Lucius, UTB, 2000.
} 
Mais il peut également contribuer à une réduction au moins momentanée de la complexité. En effet, la différenciation des sociétés modernes attribue à chaque individu différents rôles et fonctions qui entraînent une multiplication considérable de normes spécifiques de chaque domaine et de prises de positions qui en découlent. Ainsi, un homme politique doit peut-être défendre la position de son parti quant à la nécessité de réformes sociales, mais en tant que délégué local il est éventuellement de l'avis contraire, car il voit quelles conséquences de telles réformes auraient par exemple pour les plus démunis. En sa qualité de père de famille, par contre, il opterait plutôt pour une réforme des retraites, car celles-ci pèseront lourdement sur ses enfants. Si les différentes positions sont contradictoires et s'il n'est plus possible de " négocier » entre elles, le mensonge peut temporairement dissimuler ces contradictions internes et accorder à l'individu un délai qui lui permettra de trouver plus tard une attitude cohérente. Le mensonge, tout en masquant pendant un temps la contradiction entre des normes et des prises de positions différentes, sert alors - paradoxalement au même titre que la confiance - à réduire et à camoufler la multiplicité des valeurs et des normes, et contribue, certes de manière fictive, à la cohésion sociale.

Mais si la variété de ces «convictions» «fonctionnelles» et imposées ne peut plus être réduite grâce à une négociation interne de l'individu avec luimême et s'il refuse de surcroît de cacher ce conflit par un mensonge, il peut essayer de se mentir à lui-même. Or, ce type de mensonge constitue un problème théorique primordial, car si le mensonge consiste en une divergence dissimulée entre opinion et expression, il faudrait que l'individu occupe deux rôles en même temps, qu'il soit en même temps menteur et victime. Il s'ensuit que, dans ce cas, le menteur doit se dissimuler à lui-même sa véritable opinion tout en prétextant - encore une fois vis-à-vis de lui-même - qu'il en a une autre. On pourrait évidemment, pour résoudre le paradoxe, avoir recours ici à la psychanalyse et à la théorie freudienne du subconscient. Mais, comme Jean-Paul Sartre le souligne à juste titre dans le chapitre de L'Être et le Néant consacré à la mauvaise foi, cela ne saurait apporter une solution car, à ce moment-là, nous sommes confrontés à un autre paradoxe, celui d'un menteur inconscient de son mensonge ${ }^{46}$. Sartre insiste donc sur le fait que celui qui se ment à lui-même doit, lui aussi, être parfaitement conscient de son mensonge, sans pour autant expliquer davantage comment cela serait possible. Mais la conception de l'« individu » comme « dividu » réel que nous avons esquissée plus haut pourrait aider à résoudre ce paradoxe. Dans cette perspective, l'idée est concevable qu'une identité sociale liée à une certaine fonction ou position sociale dissimule à une autre sa prise de position particulière, de manière que la partie qui ment reste parfaitement consciente de la différence entre conviction et expression.

\footnotetext{
${ }^{46}$ Jean-Paul SARTRe, L'Être et le Néant. Essai d'ontologie phénoménologique, Paris, Gallimard (coll. « Tel»), 1976, p. 85.
} 
Sans le mensonge à soi-même, on ne saurait expliquer d'autres types de mensonges qui jouent un rôle considérable dans la société. En particulier, il serait impossible de saisir la collusion particulière qui peut s'installer entre le menteur et sa victime, par exemple quand un partenaire trompe l'autre "apparemment" sans que celui-ci s'en rende compte. Une telle collusion serait inconcevable si les victimes ne se mentaient pas à elles-mêmes. Cela rend possible une forme de communication où les deux instances du mensonge collaborent de manière cachée pour sa réussite. Si cette collaboration s'étend à une collectivité ou bien à une nation, il peut s'instaurer un mensonge structurel, qui consiste en la coopération permanente entre un ou plusieurs menteurs institutionnalisés d'une part, et ceux qui croient les mensonges tout en se mentant en partie à eux-mêmes de l'autre. Le cas le plus connu de ce type de collusion est évidemment celui des promesses électorales. On peut se demander pourquoi des électeurs intelligents croient chaque fois de nouveau à des promesses si souvent répétées de baisse du chômage, de réduction des impôts ou d'augmentation du pouvoir d'achat, alors que celles-ci ont déjà été démenties après les élections précédentes et que la situation économique rend fort improbable leur réalisation. Ce n'est compréhensible que si une partie de l'électorat est capable de se mentir à elle-même pour dissiper les doutes concernant les possibilités de tenir ce genre de promesses. Le gain d'un tel mensonge à soi-même est évident, car il permet à l'électeur de ne pas devoir prendre en considération la possibilité de sacrifices personnels, avec l'avantage supplémentaire de se faire plaindre comme quelqu'un qui a été trompé et trahi, tout en agissant de bonne foi. Les plaintes habituelles portées contre « les hommes politiques menteurs » font partie intégrante de ce jeu et montrent bien que la critique elle-même peut appartenir à une structure mensongère : si elle dénonce le mensonge de certains hommes politiques, elle garantit en même temps la continuité d'une collusion entre élus et électeurs qui peuvent, lors des prochaines élections, «tomber» dans le même piège. Par ailleurs, dénoncer quelques mensonges et s'en scandaliser permet en même temps le maintien du mensonge au niveau général, dont ils ne sont que des cas particuliers. Dans le contexte de ce mensonge structurel, les délimitations peuvent parfois être floues entre le mensonge à soi-même et le mensonge tout court. Il est tout à fait imaginable qu'un électeur ne se mente pas à lui-même quand il croit à des promesses tant de fois démenties, mais qu'il feigne simplement de le faire. Dans ce cas-là, la situation serait parfaitement symétrique et le mensonge serait partagé par tous les partenaires de la communication politique. Les mensonges des hommes politiques qui font des promesses qu'ils considèrent eux-mêmes comme irréalisables se trouveraient dans une harmonie préétablie avec les mensonges des électeurs qui feignent d'y croire, à tel point que la dénonciation de quelques menteurs particuliers n'est pas seulement complètement compatible avec ce système, mais qu'il en garantit même l'homéostasie. Cette structure particulière du mensonge structurel nous indique déjà en partie une réponse à la question 
posée au début, à savoir pourquoi une société ou une culture condamnent d'une manière si catégorique ce que tout le monde semble pratiquer.

\section{Les fonctions de la condamnation catégorique du mensonge}

Une raison souvent citée pour la condamnation catégorique du mensonge est que le mensonge détruirait la confiance entre des personnes de manière radicale et permanente, comme le dit bien le proverbe allemand : «Wer einmal lügt, dem glaubt man nicht, und wenn er auch die Wahrheit spricht $^{47}$. $\gg$ Ainsi, selon cette idée très répandue, il ne faut jamais mentir, même dans des situations où cela peut de prime abord paraître légitime. Dans le débat déjà évoqué avec B. Constant, Kant a présenté une version plus philosophique de cet argument général. Dans une critique de la position kantienne, Constant défend le droit de mentir dans la situation déjà évoquée par saint Augustin. Si des assassins poursuivent une victime, nous avons le droit de refuser de dire la vérité et de mentir, car selon Constant personne n'a droit à une vérité qui nuirait à autrui ${ }^{48}$. Contre cette position, Kant rectifie d'abord une erreur de Constant qui suppose que nous pouvons délibérément disposer de la vérité d'un énoncé car, en réalité, nous pouvons toujours nous tromper. En revanche, nous pouvons décider de dire ce que nous croyons ${ }^{49}$. Nous avons donc, par notre volonté, une emprise non sur la vérité, mais sur notre véracité. Après cette mise au point, Kant précise que personne n'a de droit à la véracité d'autrui, mais seulement à sa propre véracité. Et pour lui, ce droit-là n'est pas un droit personnel ou individuel, mais un droit de l'humanité entière, que personne n'a le droit de sacrifier pour des intérêts personnels. La raison que Kant donne pour cette thèse est fondée sur une théorie de la véracité comme condition sine qua non du droit.

Wahrhaftigkeit in Aussagen, die man nicht umgehen kann, ist formale Pflicht des Menschen gegen jeden, es mag ihm oder einem anderen daraus auch noch so großer Nachteil erwachsen; und, ob ich zwar dem, welcher mich ungerechterweise zur Aussage nötigt, nicht unrecht tue, wenn ich sie verfälsche, so tue ich doch durch eine solche Verfälschung, die darum auch (obzwar nicht im Sinne der Juristen) Lüge genannt werden kann, im wesentlichsten Stücke der Pflicht überhaupt Unrecht : d.i. ich mache, soviel an mir ist, dass Aussagen überhaupt keinen Glauben finden, mithin auch alle Rechte, die auf Verträgen gegründet werden, wegfallen und ihre Kraft einbüßen ; welches ein Unrecht ist, welches der Menschheit überhaupt zugefügt wird. Die Lüge also, bloß als vorsätzlich unwahre Aussage gegen einen anderen Menschen definiert, bedarf nicht des Zusatzes, dass sie einem anderen schaden müsse ; wie die Juristen es zu ihrer Definition verlangen. Denn sie schadet jederzeit einem anderen,

\footnotetext{
${ }^{47}$ L'équivalent français de cette expression serait à peu près : « Qui trop souvent crie au loup se fera dévorer. ».

${ }^{48}$ Benjamin CONSTANT, Des réactions politiques, Seconde édition, s.1., 1796-1797, p. 75.

${ }^{49}$ KANT, « Über ein vermeintes Recht », p. 35 sq.
} 
wenngleich nicht einem anderen Menschen, so doch der Menschheit überhaupt, indem sie die Rechtsquelle unbrauchbar macht. $[\ldots]^{50}$

Selon Kant, un mensonge détruit toute confiance en la véracité des affirmations et rend de ce fait une véritable communication impossible. En particulier, un mensonge invalide tous les contrats, car selon lui, ceux-ci ne seraient concevables sans l'obligation pour chacun à la véracité. La source du droit même serait dès lors invalidée. Kant part donc de l'idée que nous ne pouvons croire les déclarations des autres que si nous présupposons qu'elles sont toujours véridiques. Mais est-ce que cela correspond vraiment à notre expérience vécue ? En réalité, nous jugeons les mensonges de notre entourage non pas de manière catégorique, mais toujours en fonction des objectifs qu'il veut atteindre. Quand par exemple un ami nous dit que notre nouveau costume nous va très bien et quand nous apprenons plus tard qu'il nous a menti, c'est son dessein de nous épargner une déception ou de nous donner confiance en notre apparence qui détermine notre jugement. Mais même si, dans ce cas-là, nous condamnerions son mensonge, cela ne veut pas dire que notre confiance en cet ami est détruite à jamais. Nous ne lui ferons peut-être plus confiance pour des questions de style vestimentaire, mais cette réticence ne nous empêchera pas de nous fier à lui dans d'autres situations, comme par exemple quand il nous fait la promesse de nous prêter de l'argent. Donc, ce que Kant exclut d'emblée est une faculté humaine qui lui est par ailleurs très chère : c'est la faculté de juger chaque cas particulier qui nous permet de faire la part des choses et de décider de la crédibilité de quelqu'un ou si nous pouvons, dans une situation concrète, faire confiance à quelqu'un qui nous a peut-être menti dans une autre. Donc, un mensonge n'abolit nullement toute confiance en la crédibilité d'autrui. En fait, le manque notoire, chez Kant, d'un appel à la faculté de jugement fait apparaître un avantage considérable de toute condamnation catégorique du mensonge : il permet de renoncer à un examen détaillé des cas de figures concrets, des fonctions et des objectifs différents du mensonge et donc de réduire sa complexité à un schéma unique. Si l'on peut condamner le mensonge en tant que tel et en soi, nul besoin pour le théoricien de différencier et nul besoin non plus pour le praticien d'utiliser sa faculté de jugement. La condamnation catégorique du mensonge permettrait donc à chacun de faire l'économie d'un effort de jugement individuel et de se laisser aller à une paresse intellectuelle.

Un autre argument pour une condamnation catégorique du mensonge provient de la théorie du langage. Dans sa critique du mensonge, saint Augustin s'était servi d'un argument comparable à celui de Kant. Mais à la différence de Kant qui puise ses arguments surtout dans la philosophie du droit, les arguments de saint Augustin relèvent de la philosophie du langage, soutenue par une argumentation théologique. Augustin part du principe que Dieu a créé le langage pour permettre aux hommes de se communiquer mutuellement leurs pensées. Celui qui utilise le langage pour tromper autrui

${ }^{50}$ Ibid. 
dénature le langage et en abuse : "Celui qui utilise le langage pour tromper et non pas dans un dessein pour lequel il a été créé, commet un péché $»^{51}$. La thèse de saint Augustin fait preuve d'un réductionnisme qui a longtemps hypothéqué le discours occidental sur le mensonge tout en le doublant d'un jugement moral négatif. En fait, un simple regard sur l'usage que nous faisons du langage dans la vie quotidienne suffit pour se rendre à l'évidence : loin de servir uniquement à nous communiquer mutuellement nos véritables pensées, il peut servir des objectifs multiples. Grâce à lui, nous pouvons tromper un adversaire, attaquer quelqu'un verbalement, ouvrir un colloque, nommer quelqu'un professeur, déclarer un couple mari et femme, nous divertir ou divertir un public. C'est surtout la théorie pragmatique des actes de langage d'un Ludwig Wittgenstein ou d'un John L. Austin qui a débarrassé la philosophie du langage du réductionnisme affirmatif. Sur cette base, la théorie pragmatique aurait pu contribuer à une prise en compte de la variété des fonctions linguistiques dans toute son ampleur et par là-même à une description neutre et «par-delà le bien et le mal » de la structure linguistique du mensonge. En ce sens, Ludwig Wittgenstein avait constaté dans les Investigations philosophiques: " Le mensonge est un jeu de langage qui doit être appris comme un autre ${ }^{52}$. $\gg$ Mais contrairement à ce que l'on pourrait attendre, la théorie des actes de langage fait montre de la même réticence visà-vis du mensonge que les théories précédentes. Pour Austin par exemple, le mensonge est un acte de langage $"$ malencontreux $»^{53}$. Toutefois, cette caractérisation du mensonge ne serait valable que si le menteur avait voulu faire une déclaration sincère. Or, ce n'est évidemment pas le cas. Tout au contraire, un menteur ne rate pas, mais réussit pleinement son acte linguistique, si son interlocuteur veut bien le croire. S'il contrevient, certes, à la règle de la sincérité, il maîtrise néanmoins parfaitement les règles qui établissent l'acte linguistique de mentir.

Il n'est donc point possible de puiser dans une théorie pragmatique des arguments contre le mensonge. Néanmoins, un argument particulier peut nous aider à analyser une fonction de la condamnation catégorique du mensonge qui n'a pas encore été évoquée jusqu'à maintenant. Il s'agit d'un argument contre le mensonge utilisé, entre autres, par saint Augustin. Selon ce raisonnement, le mensonge est une contradiction en soi car le menteur présuppose chez le destinataire une croyance en la sincérité de la communication qu'il détruit lui-même ${ }^{54}$. De cette manière, tout menteur

\footnotetext{
${ }^{51}$ Aurelius Augustinus, Enchiridion de Fide, Spe et Charitate, liber unus, in S. Aurelii Augustini Opera omnia - editio latina, document online, http://www.santagostino.it/latino/enchiridion/ index.htm [12/03/2014], cité d'après la traduction allemande in Paul Kesling, Einführung, in AUgustinUs, Lüge, p. I - IL, ici p. XXVIII.

52 « Das Lügen ist ein Sprachspiel, das gelernt sein will, wie jedes andere. » (Ludwig WitTgenstein, Philosophische Untersuchungen, Frankfurt, Suhrkamp, 1971, p. 141).

53 John L. Austin, How to Do Things With Words, Oxford/ London, Oxford University Press, 1975 , p. 50.

${ }^{54}$ Augustinus, De doctrina christiana, I, 36, 40, cité par Paul KESELING, « Einführung », in AUGUSTINUS, Lüge, p. VI-XLVIII, ici p. XXII.
} 
détruirait les conditions de possibilité de son propre acte. Pour des raisons d'espace et de temps, nous ne sommes pas en mesure de discuter ici en détail le bien-fondé de cette thèse et nous devons nous contenter de renvoyer à un argument déjà avancé. Certes, un menteur présuppose chez son interlocuteur la croyance en la véracité de ce qu'il dit et s'il veut que son mensonge réussisse, il doit tout faire pour lui paraître crédible. Mais cette croyance ne s'étend pas automatiquement à tous les énoncés et un mensonge dans un cas particulier ne détruit point nécessairement la croyance en la sincérité en général. Si nous apprenons par hasard que quelqu'un nous a menti dans une situation concrète, nous n'en concluons nullement de ne plus jamais le croire, mais nous serons peut-être plus prudents dans des situations futures comparables à celle du premier mensonge.

Toutefois, ce qui est faux sur le plan logique l'est beaucoup moins sur le plan pragmatique. Pour que le mensonge puisse fonctionner, le menteur doit faire en sorte que son interlocuteur croie en sa sincérité. Or, toute affirmation de sincérité a le même effet paradoxal qu'un démenti : il provoque le soupçon qu'il veut dissiper. Si quelqu'un insiste sans raison apparente sur sa véracité, nous sommes plutôt enclins à mettre en doute ce qu'il dit. Le menteur ne saurait donc inspirer de manière directe la croyance en sa bonne foi, dont il a cependant besoin pour que le mensonge puisse réussir. Mais le défaut de moyens directs pour augmenter la crédibilité ne veut pas dire qu'il n'en existe pas du tout. En effet, on peut renforcer sa crédibilité d'une manière indirecte en dénonçant le mensonge. Le procédé consiste en l'inversion du soi-disant paradoxe du mensonge. Si, selon la critique classique, tout menteur détruit la croyance en la sincérité sans laquelle son mensonge ne pourrait fonctionner, on peut conclure que le meilleur moyen pour faire croire à sa propre sincérité est une condamnation sans faille et catégorique du mensonge. Ce serait donc la condamnation du mensonge qui crée l'impression d'une sincérité inconditionnelle et fait en sorte que le mensonge peut mieux fonctionner. Évidemment, une telle technique connaît ses limites sur le plan individuel, car elle ne saurait marcher que jusqu'au moment où le menteur est découvert. Il s'ensuivrait une perte de crédibilité radicale de celui-ci. Mais ces limites n'existent pas si nous étendons notre raisonnement à des collectivités comme une société ou une culture. En effet, à ce niveau-là, la condamnation catégorique du mensonge, indépendamment des objectifs et effets particuliers, concourt d'une manière considérable à son meilleur fonctionnement. Dans cette perspective, la condamnation catégorique dans le domaine de la morale contribuerait à son fonctionnement dans la pratique quotidienne. Ainsi, le mensonge présupposerait un méta-mensonge ou mensonge sur le mensonge, par lequel une société ou une culture s'aveugle sur sa propre pratique.

Au début de nos réflexions sur le mensonge, nous nous sommes demandé si la condamnation véhémente et catégorique d'une pratique aussi répandue dans une société ou une culture obéit à une raison qui ne relèverait pas de la divergence classique entre morale et pratique, mais qui serait spécifique du 
mensonge. Il se pourrait que ce motif spécifique se trouve dans cette interprétation de la condamnation catégorique du mensonge comme une manifestation de sincérité collective. À ce propos, ce n'est probablement pas le fruit du pur hasard si la condamnation la plus radicale du mensonge et la tentative la plus dure et cruelle de forcer des êtres humains à la véracité par tous les moyens, y compris la torture, la menace de mort ou bien de condamnation éternelle, se trouvent justement dans des institutions autocratiques ou totalitaires qui se croient en possession d'une vérité absolue et qui sont en réalité mensongères. L'histoire de l'Inquisition et de l'Église catholique, du KGB et du communisme, de la Gestapo et du fascisme en dit long. Si l'on passe en revue cette histoire impressionnante du mensonge totalitaire, on ne saurait se soustraire au soupçon que la condamnation morale et catégorique du mensonge est peut-être la condition de possibilité de son meilleur fonctionnement. 